\title{
Urgência e descontinuidade do cuidado em saúde bucal de crianças e adolescentes
}

\author{
Urgency and discontinuity of oral health care in children and \\ adolescents
}

Fábio Augusto de Oliveira Pereira (https://orcid.org/0000-0002-1441-4226) ${ }^{1}$

Luciana Reichert da Silva Assunção (https://orcid.org/0000-0002-7380-8583) ${ }^{1}$

Fernanda Morais Ferreira (http://orcid.org/0000-0001-9400-1167) ${ }^{2}$

Fabian Calixto Fraiz (https://orcid.org/0000-0001-5290-7905) ${ }^{1}$
${ }^{1}$ Departamento de Estomatologia, Universidade Federal do Paraná. Av. Prefeito Lothário Meissner 632, Jardim Botânico. 80210-170 Curitiba PR Brasil.fabio_opereira@ hotmail.com

${ }^{2}$ Departamento de Pediatria e Ortodontia, Universidade Federal de Minas Gerais. Belo Horizonte Minas Gerais Brasil.

\begin{abstract}
The aim of the present study was to investigate the discontinuity of oral health care among children and adolescents who accessed emergency services at primary care units and urgent care units. Records were reviewed of patients aged 0 to 17 years treated in the public healthcare system in city of Curitiba, Brazil. Discontinuity was considered when elective treatment was not registered at the primary care unit within six months after urgent care. The sample was stratified based on the Municipal Human Development Index (MHDI) $(\leq 0.799$ or $\geq 0.800)$. The association between discontinuity and covariables was assessed using univariate and multivariate Poisson regression models with robust variance $(\alpha=0.05)$. The incidence of discontinuity was $42.2 \%$. In the MHDI $\leq 0.799$ stratum, the risk of discontinuity was greater among children younger than five years of age and individuals who had not had a dental appointment in the previous year. In both MHDI strata, the risk of discontinuity was higher in patients who received urgent care at an urgent care unit than those treated at a primary care unit. The incidence of the discontinuity of oral health care was high and was strongly influenced by the characteristics of dental service utilization among individuals living in regions with a lower MHDI.
\end{abstract}

Key words Continuity of Patient Care, Oral health, Unified Health System, Child, Adolescent
Resumo Este trabalho estudou a descontinuidade do cuidado (DC) em saúde bucal entre crianças e adolescentes que acessaram os serviços de urgência nas unidades básicas de saúde (UBS) e de pronto atendimento (UPA). Foram avaliados prontuários de indivíduos de 0 a 17 anos vinculados ao Sistema Único de Saúde de Curitiba. Considerou-se DC quando não houve o registro de consulta eletiva na UBS nos 6 meses subsequentes ao atendimento de urgência. A amostra foi estratificada pelo Índice de Desenvolvimento Humano Municipal (IDHM) da Unidade de Desenvolvimento Humano em que o paciente residia em $\leq 0,799$ ou $\geq 0,800$. A associação entre DC e demais covariáveis foi analisada através de regressão de Poisson com variância robusta uni e multivariada $(\alpha=0,05)$. A incidência de DC foi de $42,2 \%$. No estrato de IDHM $\leq 0,799$, o risco de DC foi maior para as crianças com menos de 5 anos de idade e aqueles que não visitaram o dentista no último ano. Nos dois estratos, o risco de DC foi maior para aquelas que utilizaram a UPA para o atendimento de urgência quando comparadas com as que receberam atendimento na UBS. A DC em saúde bucal apresentou alta incidência, sendo que entre os residentes em regióes com menor IDHM, sofreu uma maior influência das características de uso dos serviços.

Palavras-chave Continuidade da Assistência ao Paciente, Saúde Bucal, Sistema Único de Saúde, Criança, Adolescente 


\section{Introdução}

Sistemas de saúde que se organizam através da Atenção Primária à Saúde (APS) apresentam melhores indicadores de saúde, equidade de acesso e custos mais baixos ${ }^{1}$. A APS é o primeiro nível de atenção no sistema de saúde e suas ações devem ser humanizadas e centradas no indivíduo, abordando a família e orientada para comunidade. Caracteriza-se, também, pela resolutividade e integração, acompanhamento do indivíduo ao longo do tempo e como porta preferencial de acesso ao sistema de saúde ${ }^{2}$. Estes aspectos tornam possível a construção de uma relação estável, personalizada e de longa duração entre a população e os profissionais de saúde ${ }^{3}$.

No sistema de saúde pública brasileiro, o Sistema Único de Saúde (SUS), a APS é estruturada através de uma rede de equipamentos de saúde, denominados de Unidades Básicas de Saúde (UBS), que situam-se próximos à residência dos indivíduos. As UBS se apresentam como fonte regular de cuidados primários à saúde $\mathrm{e}^{4}$ devem ser o principal ponto de contato entre os usuários e o sistema de saúde. Nesse sistema, para os atendimentos dos casos de saúde emergenciais, existem as Unidades de Pronto Atendimento (UPA), que junto com as UBS e outros locais de atendimento, compõem a rede de atenção à urgência e emergência ${ }^{5}$.

No Brasil, as doenças crônicas representam a maior parte da carga de doença, impactando significativamente no Sistema Único de Saúde (SUS $^{6}$. Esses agravos, cada vez mais prevalentes na população devido à transição demográfica, compartilham dos mesmos fatores de risco, associados ao estilo de vida, hábitos alimentares, uso de tabaco, consumo excessivo de álcool e padrões de higiene. Hipertensão, diabetes e doenças cardiovasculares e, inclusive, doenças bucais, fazem parte deste tipo de condição ${ }^{7}$ e podem, durante seu transcurso, apresentar processos de agudização.

Um importante desfecho em saúde bucal que poderia ser utilizado pelos sistemas de saúde, como um claro indicador de saúde na população, é a ausência de dor/desconforto de origem den$\mathrm{tal}^{8}$. A literatura tem demonstrado que a qualidade de vida ${ }^{9,10}$ e a percepção da saúde bucal de crianças e adolescentes são fortemente impactados por episódios de dor de dente ${ }^{8,11}$. Esta condição, também, pode gerar transtornos aos pais/ responsáveis, como o absenteísmo ao trabalho ${ }^{12}$. No Brasil, apesar dos avanços dos últimos anos na área de saúde bucal coletiva ${ }^{13}$, a prevalência de dor de dente ainda atinge uma parcela significativa da população infantil ${ }^{14}$ e de adolescentes ${ }^{15}$. Além disso, alguns estudos verificaram que regiões com piores índices de desenvolvimento humano (IDH) apresentam mais relatos de dor de dente entre crianças e adolescentes ${ }^{14,15}$.

Assim, considerando a alta prevalência de dor odontogênica e a necessidade de prevenir novos eventos, é importante que os equipamentos da APS estejam organizados para propiciar a continuidade do cuidado após a resolução da urgência em saúde bucal. Já que a utilização regular de serviços de saúde bucal, especialmente de serviços preventivos, tem sido associada a uma menor utilização de serviços de urgência ${ }^{16}$.

Apesar da relevância do tema e do conhecimento dos fatores associados à utilização dos serviços odontológicos de urgência, principalmente em universidades e/ou em departamentos de emergência hospitalares ${ }^{17-19}$, ainda faltam pesquisas que avaliem a continuidade do cuidado na atenção regular de crianças e adolescentes atendidas na urgência odontológica do sistema público de saúde.

Como o relato de dor de dente é motivado principalmente por agravos passíveis de prevenção e tratamento na APS, e esta, por sua vez, é o nível de atenção do sistema de saúde responsável pelo acompanhamento dos indivíduos, o objetivo deste estudo foi analisar os fatores associados à descontinuidade do cuidado em saúde bucal na UBS de crianças e adolescentes que receberam atendimento de urgência em UBS e UPA de Curitiba.

Um melhor entendimento desses fatores poderá auxiliar gestores públicos na organização de uma rede de saúde bucal que vise o estabelecimento de vínculo entre crianças e adolescentes atendidos na urgência odontológica e no sistema de saúde.

\section{Métodos}

\section{Desenho do estudo e população estudada}

Estudo do tipo prospectivo no qual foram selecionados todos os prontuários eletrônicos de indivíduos da faixa etária entre zero e 17 anos que acessaram serviços de urgência nos equipamentos de saúde bucal municipal de Curitiba no mês de abril de 2014. Os atendimentos à urgência em saúde bucal em Curitiba são realizados em 108 Unidades Básicas de Saúde (UBS) e três Unidades de Pronto Atendimento (UPA). 
Apenas indivíduos com cadastro definitivo no sistema de prontuários eletrônicos (e-saúde) da Secretaria Municipal de Saúde (SMS) de Curitiba foram incluídos no estudo.

\section{Coleta de dados e aspectos éticos}

Os dados foram obtidos por um único pesquisador a partir do sistema de prontuários eletrônicos chamado $e$-saúde. Esse sistema inclui informações de toda a rede de saúde bucal integrante da Atenção Primária à Saúde (APS) e da rede de urgência e emergência da SMS de Curitiba. Considerou-se como descontinuidade do cuidado a ausência de registro de atendimento odontológico eletivo na UBS nos seis meses subsequentes ao atendimento de urgência. Para cada indivíduo atendido na urgência foram obtidas dos prontuários eletrônicos as seguintes informações: sexo, idade, UBS de cadastro, regional administrativa de cadastro, ocorrência de atendimento anterior na rede municipal de saúde bucal, tipo de atendimento da última consulta odontológica, data da última visita no dentista, tipo de equipamento de saúde utilizado para o atendimento de urgência.

A amostra foi estratificada de acordo com o índice de desenvolvimento humano municipal (IDHM) da Unidade de Desenvolvimento Humano (UDH) da residência do paciente. O IDHM é o ajuste, ao nível municipal, do índice de desenvolvimento humano, que, como este último, avalia as dimensões de renda, educação e longevidade na população. $\mathrm{O}$ seu valor pode variar entre 0 e 1 , sendo quanto mais próximo de 1, melhor a condição apresentada na população. Nas regiões metropolitanas, o IDHM é atribuído a recortes espaciais intrametropolitanos que apresentam características socioeconomicamente mais homogêneas, chamadas de unidades de desenvolvimento humano (UDH $)^{20}$. Segundo os últimos dados do Programa das Nações Unidas para o Desenvolvimento (PNUD) de 2013, os valores dos IDHM das UDH do município de Curitiba variaram entre 0,62 a $0,93^{20}$. O valor do IDHM da respectiva UDH da residência do atendido foi obtido através do mapa para o desenvolvimento humano, disponível no endereço eletrônico: http://www.atlasbrasil.org.br/2013/, sendo utilizado para a busca o endereço da UBS de cadastro do indivíduo.

Este estudo foi submetido e aprovado pelo Comitê de Ética em Pesquisa do Setor de Ciências da Saúde da Universidade Federal do Paraná.

\section{Análise Estatística}

Os dados coletados foram tabulados e analisados através do programa SPSS ${ }^{\circledR}$ Statistics (IBM Corp. Released 2017. IBM SPSS Statistics for Windows, Versão 25.0. Armonk, NY: IBM Corp). A associação entre a descontinuidade do cuidado e as demais covariáveis foi analisada através de regressão de Poisson com variância robusta univariada e multivariada. $\mathrm{O}$ risco relativo, com o seu respectivo intervalo de confiança de $95 \%$ foram obtidos com as análises. Foram incluídas no modelo de regressão multivariado as variáveis que apresentavam um valor de $\mathrm{p}<0,20$ na análise univariada. As variáveis "Tipo do último atendimento odontológico" e "Visitou o dentista no último ano”, não foram incluídas no modelo, pois são subgrupos de uma das categorias da variável " 1 ' acesso ao SUS/Saúde bucal". O nível de significância adotado foi de $5 \%$.

Para a análise estatística foram dicotomizadas as variáveis: tipo do último atendimento odontológico (eletivo e urgência), tipo de equipamento utilizado na urgência odontológica (UPA e UBS). A idade foi categorizada em três faixas etárias ( $\leq 04$ anos, 05 até 11 anos e $\geq 12$ ). Para definir a ocorrência de atendimento anterior na rede municipal de saúde bucal foi considerado se $o$ atendimento de urgência era o primeiro acesso ou não. Verificou-se se a criança/adolescente havia visitado o dentista no SUS/Curitiba no último ano (sim e não). A descontinuidade do cuidado na UBS, variável desfecho, foi verificada através da ausência de algum registro no prontuário eletrônico para uma consulta programada ou de avaliação inicial no período de seis meses subsequentes ao atendimento de urgência. A população estudada foi estratificada em dois grupos de acordo com o valor do IDHM da UDH da residência do paciente e classificado conforme o Programa das Nações Unidas para o Desenvolvimento em: IDHM $\leq 0,799$ e IDHM $\geq 0,800$ (PNUD, 2013).

\section{Resultados}

Foram avaliados 1.012 prontuários eletrônicos de crianças e adolescentes com até 17 anos de idade que receberam atendimento de urgência na rede municipal de atenção à saúde bucal de Curitiba no mês de abril de 2014, sendo 908 nas UBS e 104 nas UPA. Da amostra total, 66,8\% e $33,2 \%$ residiam em UDHs com IDHM $\leq 0,799$ e IDHM $\geq 0,800$, respectivamente (Tabela 1 ). A 
Tabela 1. Características dos participantes. CuritibaPR, 2014 ( $\mathrm{N}=1.072)$.

\begin{tabular}{|c|c|c|c|}
\hline \multicolumn{2}{|c|}{ Variáveis } & \multirow{2}{*}{$\begin{array}{c}\text { Freq } \\
480\end{array}$} & \multirow{2}{*}{$\begin{array}{c}\% \\
47,4\end{array}$} \\
\hline Gênero & Masculino & & \\
\hline \multirow{4}{*}{ Faixa etária } & Feminino & 532 & 52,6 \\
\hline & $\leq 04$ anos & 174 & 17,2 \\
\hline & 05 a 11 anos & 516 & 51,0 \\
\hline & $\geq 12$ anos & 322 & 31,8 \\
\hline \multirow{2}{*}{$\begin{array}{l}1^{\circ} \text { Acesso ao } \\
\text { SUS/Saúde Bucal }\end{array}$} & Sim & 123 & 12,2 \\
\hline & Não & 889 & 87,8 \\
\hline \multirow{2}{*}{$\begin{array}{l}\text { Tipo de } \\
\text { equipamento }\end{array}$} & UPA & 104 & 10,3 \\
\hline & UBS & 908 & 89,7 \\
\hline \multirow{2}{*}{$\begin{array}{l}\text { Tipo do último } \\
\text { atendimento }\end{array}$} & Urgência & 97 & 10,9 \\
\hline & Eletivo & 792 & 89,1 \\
\hline \multirow{2}{*}{$\begin{array}{l}\text { Visitou o dentista } \\
\text { no último ano }\end{array}$} & Não & 282 & 68,3 \\
\hline & Sim & 607 & 31,7 \\
\hline \multirow{2}{*}{$\begin{array}{l}\text { IDHM da UDH } \\
\text { de residência }\end{array}$} & $\geq 0,800$ & 336 & 33.2 \\
\hline & $\leq 0,799$ & 676 & 66,8 \\
\hline \multicolumn{4}{|c|}{$\begin{array}{l}\text { **Considerado apenas os que já haviam recebido atendiment } \\
\text { odontológico na rede de atenção à saúde bucal da Secretaria } \\
\text { Municipal de Saúde de Curitiba. Freq= Frequência; IDHM } \\
\text { = Índice de Desenvolvimento Humano Municipal; UDH = } \\
\text { Unidade de desenvolvimento Humano. }\end{array}$} \\
\hline
\end{tabular}

idade média dos indivíduos foi de 9 anos, 5 meses e 26 dias (Desvio padrão $=4,7$ ).

A taxa de incidência de DC foi de $42,2 \%$ da amostra total. Os grupos que residiam em UDHs com IDHM $\leq 0,799 \mathrm{e} \geq 0,800$ apresentaram incidências de DC muito próximas, $43,3 \%$ e 39,8\%, respectivamente.

A distribuição de frequência por sexo na população de estudo se apresentou de maneira equilibrada, sendo $52,6 \%$, do sexo feminino (Tabela 1), não havendo diferença significativa entre o sexo e a DC em nenhum dos estratos (Tabelas 2 e 3$)$.

No grupo residente em UDHs com IDHM $\leq$ 0,799 , o risco de DC foi maior entre aqueles que não haviam visitado o dentista no último ano no SUS/Curitiba ( $\mathrm{RR}_{\mathrm{b}}: 1,44$; IC95\%:1,20-1,74) (Tabela 2). $\mathrm{O}$ fato do atendimento de urgência ser o primeiro acesso apresentou associação com a DC na análise univariada $\left(\mathrm{RR}_{b}: 137\right.$; IC95\%:1,111,69) (Tabela 2), mas perdeu a significância quando foi controlado pelas demais covariáveis (RR:1,22; IC95\%:0,97-1,53) (Tabela 4). O modelo multivariado também demonstrou que as crianças da faixa etária de 5 a 11 anos apresentaram uma menor incidência de DC ( $\mathrm{RR}_{a}: 0,74$; IC95\%:0,58-0,92) quando comparadas com aquelas que tinham 4 anos ou menos. Além disso, aquelas que receberam o atendimento de urgên- cia em UPA apresentaram um risco 34\% maior de DC do que as que receberam esse atendimento em UBSs (RR :1,34 IC95\%: 1,07-1,67) (Tabela 4).

No grupo residente em UDHs com IDHM $\geq$ 0,800 , a única variável associada a descontinuidade do cuidado foi o tipo de equipamento utilizado na urgência, sendo que aqueles atendidos em UPAs apresentaram um risco 53\% maior de descontinuidade do que aqueles atendidos em UBS (RR:1,53 IC95\%: 1,10-2,13) (Tabela 3).

\section{Discussão}

Uma importante parcela de crianças e adolescentes não apresentou continuidade do cuidado após atendimento de urgência odontológica recebido na rede de saúde bucal pública, sendo a proporção muito semelhante nos dois estratos de IDHM.

Tem sido observado que uma grande parte daqueles que utilizam serviços de urgência odontológica mais de uma vez tem maior probabilidade de continuar a utilizá-los como seu principal meio para o atendimento odontológico ${ }^{17}$. Crianças e adolescentes que buscam os serviços de saúde motivados por dor de dente e que apresentam descontinuidade estão sujeitas a novos episódios de dor e sofrimento, inclusive com impactos em suas atividades cotidianas, como ir para escola, comer, brincar, dormir ${ }^{10,11}$. O acompanhamento regular em saúde bucal deste grupo etário pelas UBS poderia, além de tratar e controlar a evolução das lesões de cárie, evitar a procura recorrente ao dentista devido a um episódio de dor.

Nesse estudo, em ambos os estratos, a procura por um serviço direcionado para o atendimento das urgências odontológicas, como o setor de saúde bucal das UPAs, foi um fator de maior risco para DC de crianças e adolescentes na atenção regular quando comparados com aqueles que receberam atendimento em UBSs.

Diversos aspectos podem estar relacionados a este achado. Deve-se considerar que os indivíduos oriundos de áreas de IDHM $\geq 0,800$ podem apresentar mais facilidades para dar sequência ao tratamento odontológico em outros locais que não a UBS, limitando-se a um atendimento eventual na UPA em momentos de urgência e indisponibilidade de outros serviços.

Embora os indivíduos com piores condições socioeconômicas procurem mais os serviços de urgência ${ }^{21}$ e encontrem mais barreiras de acesso aos serviços regulares de saúde bucal ${ }^{22,23}$, deve-se considerar que a interação, ainda pouco efetiva, entre a UPA e a UBS ${ }^{21}$ também pode estar con- 
Tabela 2. Regressão univariada de poisson com variância robusta para descontinuidade do cuidado no grupo que residia em UDH com IDHM $\leq$ 0,799. Curitiba-PR, $2014(\mathrm{~N}=676)$.

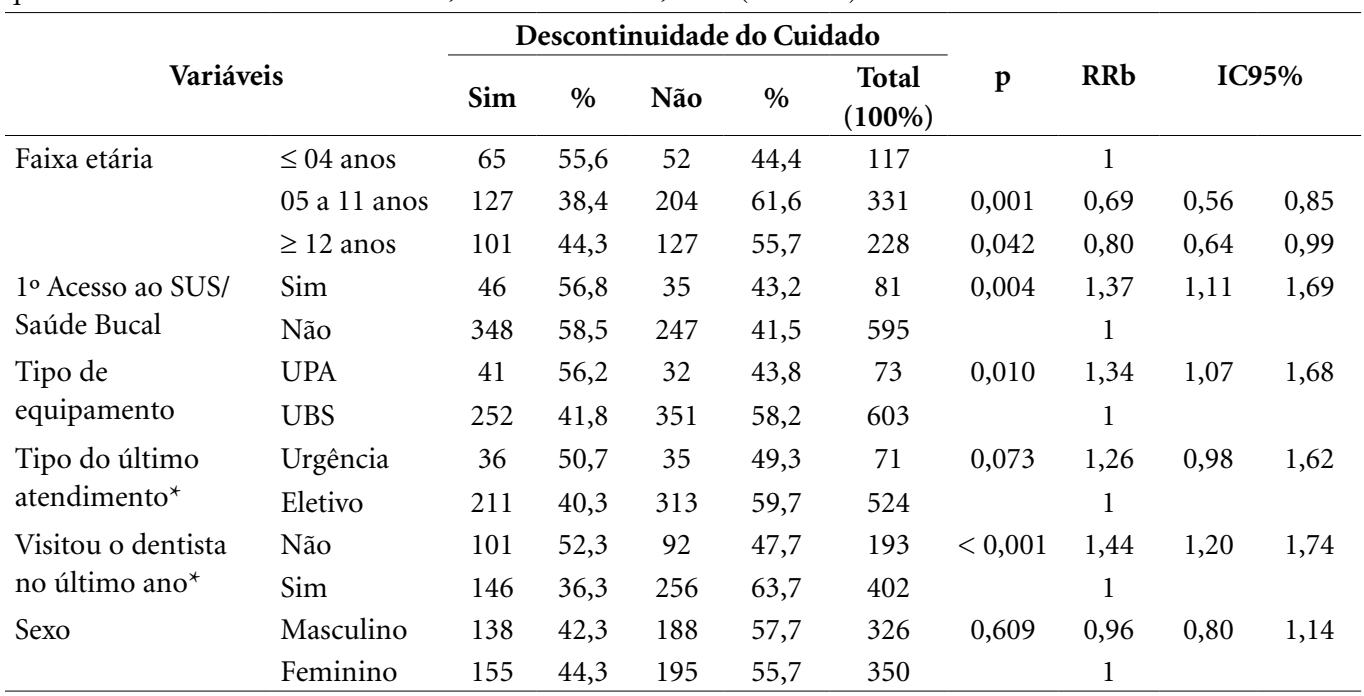

$\mathrm{RRb}=$ Risco Relativo Bruto; IC = intervalo de confiança. Resultados significantes ao nível de $5 \%$ marcados em negrito. ${ }^{*}$ Considerado apenas os que já haviam recebido atendimento odontológico na rede de atenção à saúde bucal da Secretaria Municipal de Saúde de Curitiba.

Tabela 3. Regressão univariada de poisson com variância robusta para descontinuidade do cuidado em saúde bucal no grupo que residia em UDH com IDHM $\geq 0$,800. Curitiba-PR, $2014(\mathrm{~N}=336)$.

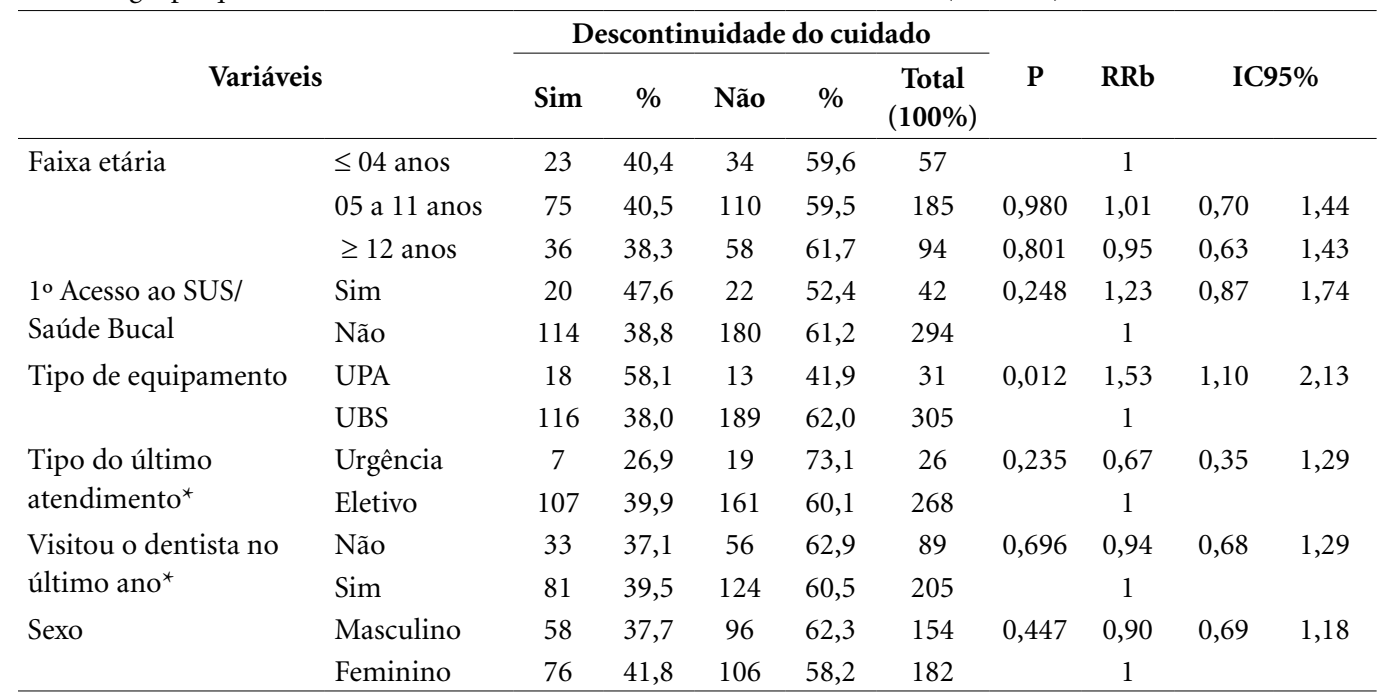

$\mathrm{RRb}=$ Risco Relativo Bruto; IC = intervalo de confiança. Resultado significante ao nível de 5\% marcados em negrito.

${ }^{*}$ Considerado apenas os que já haviam recebido atendimento odontológico na rede de atenção à saúde bucal da Secretaria Municipal de Saúde de Curitiba.

tribuindo para a DC neste grupo. Em recente revisão sobre as barreiras e os facilitadores da integração da saúde bucal na atenção primária, foi demonstrado que a descontinuidade no processo de atendimento integrado foi associada a sistemas de referência inadequados e interface deficiente entre os diversos serviços ${ }^{24}$. Espera-se que as estratégias de orientação daqueles que recebem atendimentos de urgência nas UPAs para buscarem a continuidade nas ações em saúde bucal nas UBSs, como a utilização de guias de referência e contra referência, aliadas a facilidade de agendamento e definição clara de prioridades, minimizem este problema. 
Tabela 4. Modelo de regressão multivariada de poisson com variância robusta para descontinuidade do cuidado em saúde bucal em residentes em UDH com IDHM $\leq$ 0,799. Curitiba-PR, 2014.

\begin{tabular}{|c|c|c|c|c|}
\hline \multicolumn{2}{|c|}{ Variáveis } & p & RRa & IC 95\% \\
\hline $1^{\circ}$ Acesso ao & Sim & \multirow[b]{2}{*}{0,092} & 1,22 & \multirow[t]{2}{*}{$0,97-1,53$} \\
\hline $\begin{array}{l}\text { SUS/Saúde } \\
\text { Bucal }\end{array}$ & Não & & 1 & \\
\hline \multirow{2}{*}{$\begin{array}{l}\text { Tipo de } \\
\text { equipamento }\end{array}$} & UPA & \multirow{2}{*}{0,011} & 1,34 & \multirow[t]{2}{*}{$1,07-1,67$} \\
\hline & UBS & & 1 & \\
\hline \multirow[t]{3}{*}{ Faixa etária } & $\leq 04$ anos & & 1 & \\
\hline & 05 a 11 anos & 0,008 & 0,74 & $0,58-0,92$ \\
\hline & $\geq 12$ anos & 0,118 & 0,83 & $0,65-1,05$ \\
\hline
\end{tabular}

$\mathrm{RRa}=$ Risco Relativo ajustado; IC = intervalo de confiança.

Resultados significantes ao nível de 5\% marcados em negrito.

No estudo realizado foi observado que associação entre a faixa etária e a DC apenas no estrato com menor IDHM. As crianças menores de 05 anos do grupo de IDHM $\leq 0,799$ retornavam menos na atenção regular após um atendimento de urgência odontológica. Já foi demonstrado que grande parte das crianças atendidas nos serviços de urgência não recebe qualquer tipo de procedimento clínico odontológico para alívio da dor, apenas medicações, solicitações de exames radiográficos ou, ainda, são encaminhadas para outro tipo de serviço ${ }^{19,25}$. A baixa resolutividade pode levar a um agravamento do problema apresentado inicialmente, já que, quanto mais adiado é o tratamento odontológico de crianças, mais complexo e difícil se torna a intervenção e o acesso ao serviço de saúde ${ }^{26}$. Outro aspecto, que deve ser observado é que a ausência de retorno das crianças menores para atendimento também pode tanto estar relacionada à baixa percepção dos pais sobre a importância da saúde bucal e da necessidade de consultas odontológicas regulares para seus filhos ${ }^{27}$.

Embora no município de Curitiba a maior parte da sua população, cerca de $60 \%$, resida em regiões de IDHM classificado como muito alto ( $\geq$ 0,800 ) (PNUD), no presente estudo, as crianças e adolescentes que procuraram os serviços públicos municipais de saúde bucal para um atendimento de urgência eram oriundos, em sua maioria, de áreas da cidade que apresentavam IDHM menor. Este resultado pode estar relacionado ao maior risco de crianças e adolescentes apresentarem dor de dente conforme diminui o valor do IDH da região de moradia ${ }^{14,16}$. Além disso, a busca pelos serviços do SUS é reduzida com o aumento da renda e da educação $0^{28}$, sendo estes, indicadores diretamente relacionados ao valor atribuído ao $\mathrm{IDHM}^{20}$. É provável que quanto maior o IDHM da região, menor a demanda pelos serviços do SUS, inclusive, os de urgência odontológica.

Verificou-se que, em indivíduos do grupo de IDHM $\leq 0,799$ que não visitaram o dentista no último ano, o risco de descontinuar o cuidado em saúde bucal era maior. Outro estudo, limitado a adultos estadunidenses beneficiários do programa de saúde Medicaid, encontrou resultado semelhante ${ }^{18}$. Observa-se assim, a importância da regularidade das consultas odontológicas para crianças e adolescentes. Considerando que ao nível de sistema de saúde, a adesão de crianças ao atendimento odontológico é favorecida quando existe uma política de referenciamento pelos médicos de família e pediatras para o dentista ${ }^{27}$, mudanças nos processos de trabalho que estabeleçam fluxos regulares, entre as equipes médica e de enfermagem com as equipes de saúde bucal, principalmente para aqueles sem acompanhamento regular no dentista, poderia facilitar a continuidade do cuidado na atenção à saúde bucal, inclusive para aqueles que procurassem os serviços de urgência.

A associação entre o maior risco de DC e a ausência de vínculo com a rede publica de atenção a saúde anterior à consulta de urgência entre aqueles que moravam em áreas de IDHM $\leq$ 0,799 observada na análise univariada, deixou de existir quando ajustado pelas demais covariáveis. Outras pesquisas devem ser desenvolvidas para aprofundar o entendimento do impacto do primeiro contato com o sistema público de saúde ocorrer através do atendimento de urgência. Estudos têm indicado que crianças e adolescentes, oriundos de famílias que apresentam piores condições socioeconômicas, recorrem aos serviços de saúde bucal apenas quando percebem um problema $^{22,23}$, e muitas vezes esse é o primeiro contato do paciente com o serviço odontológi$\mathrm{Co}^{17}$. Além disso, esses grupos buscam menos os serviços de saúde bucal regular ${ }^{23}$. O acolhimento, enfatizado na Política Nacional de Saúde Bucal ${ }^{29}$, pode ter um papel importante na vinculação destes indivíduos com o sistema de saúde já que muitos estão tendo a primeira experiência com os serviços em saúde. Além disso, a construção de vínculo com a equipe de saúde pode depender de quão resolutivo possa ser este primeiro contato com o serviço. A estratégia de saúde da família, além de apresentar importante impacto nos desfechos em saúde, também contribuiu para o fortalecimento desse vínculo ${ }^{30}$. 
Como todo estudo que envolve apenas dados secundários, algumas limitações foram observadas. Alguns aspectos que poderiam estar associados ao desfecho, como o nível de urgência de cada atendimento e dados demográficos dos pais não puderam ser utilizados devido às inconsistências encontradas nos registros. Outro ponto que deve ser considerado é que apenas serviços públicos foram avaliados no seguimento dos atendidos na urgência odontológica, o que não permite generalizações. Devido aos aspectos éticos não se pode utilizar o endereço residencial do atendido para determinar à respectiva UDH, utilizando-se assim o da UBS de cadastro. Entretanto, considerando que este estudo foi desenvolvido em uma grande metrópole que conta com uma rede de atenção à saúde bucal pública estruturada, onde a faixa etária estudada utiliza os serviços do SUS com grande frequência, acaba por minimizar os efeitos dessas limitações na validade dos resultados. Também deve-se considerar que, devido ao tamanho da população estudada, os dados não foram coletados em duplicata. Por fim, embora a busca por atendimento regular possa ter ocorrido em um período maior do que seis meses nesse estudo, esse foi considerado o tempo máximo para o atendimento eletivo.

Muitas crianças e adolescentes descontinuaram o cuidado em saúde bucal ao receberem um atendimento de urgência nos equipamentos públicos de saúde, principalmente quando utilizaram as UPAs. O acesso aos serviços de saúde bucal muitas vezes ocorre por meio da urgência odontológica. Os resultados deste estudo indicam a clara necessidade de ampliar as medidas e ações que estimulem a continuidade do cuidado em saúde bucal de crianças e adolescentes atendidos na urgência. Essa estratégia pode favorecer o vínculo desses indivíduos com as equipes de atenção básica, visando uma atenção à saúde integral e mais resolutiva.

\section{Colaboradores}

FAO Pereira, LRS Assunção e FC Fraiz foram responsáveis pela concepção e desenho do estudo. FAO Pereira e FC Fraiz participaram da coleta de dados. A análise e interpretação dos dados foi realizada por FAO Pereira, LRS Assunção, FM Ferreira, FC Fraiz. Todos os autores contribuíram para o arcabouço conceitual de redação e interpretação do artigo, bem como a versão final.

\section{Referências}

1. Starfield B, Shi L. Policy relevant determinants of health: an international perspective. Health Policy 2002; 60(3):201-218.

2. World Health Organization (WHO). The world health report 2008: primary health care now more than ever [Internet]. Geneva: WHO; 2008. [acessado 2016 Nov 9] Disponível em: https://www.who.int/whr/2008/en/

3. Starfield B. Atenção Primária: Equilíbrio entre necessidades de saúde, serviços e tecnologia [Internet]. Brasília: UNESCO, MS; 2002. [acessado 2016 Nov 9] Disponível em: http://bvsms.saude.gov.br/bvs/publicacoes/ atencao_primaria_p1.pdf

4. Cunha EM, Giovanella L. Longitudinalidade/continuidade do cuidado: identificando dimensões e variáveis para a avaliação da Atenção Primária no contexto do sistema público de saúde brasileiro. Cien Saude Colet 2011; 16(Supl. 1):1029-1042.

5. O'Dwyer G, Konder MT, Reciputti LP, Lopes MGM, Agostinho DF, Alves GF. The process of implementation of emergency care units in Brazil. Rev Saude Pública 2017; 51:125.

6. Mendes, EV. Interview: The chronic conditions approach by the Unified Health System. Cien Saude Colet 2018; 23(2):431-436. 
7. Organização Mundial da Saúde (OMS). Prevenção de doenças crônicas um investimento vital [Internet]. Geneva: OMS; 2005. [acessado 2016 Nov 2] Disponível em: https://www.paho.org/bra/index.php?option=com_docman\&view $=$ document\&layout $=$ default $\&$ alias $=328$-prevencao-doencas-cronicas-um-investimento-vital-8\&category_slug=doencas-cronicas $-116 \&$ Itemid $=965$

8. Shepherd MA, Nadanovsky P, Sheiham A. The prevalence and impact of dental pain in 8-year-old school children in Harrow, England. Br Dent J 1999; 187(1):38-41.

9. Freire MCM, Côrrea-Faria P, Costa RC. Effect of dental pain and caries on the quality of life of Brazilian preschool children. Rev Saude Publica 2018; 52:30.

10. Gomes MC, Neves ÉTB, Perazzo MF, Paiva SM, Ferreira FM, Granville-Garcia AF. Contextual and individual determinants of oral health-related quality of life among five-year-old children: a multilevel analysis. Peer J 2018; 6:e5451.

11. Peres KG, Cascaes AA, Leão ATT, Côrtes MLS. Aspectos sociodemográficos e clínicos da qualidade de vida relacionada à saúde bucal em adolescentes. Rev Saude Publica 2013; 47(Supl. 3):19-28.

12. Ribeiro GL, Gomes MC, Lima KC, Martins CC, Paiva SM, Granville-Garcia AF. Work absenteeism by parents because of oral conditions in preschool children. Int Dent J 2015; 65(6):331-337.

13. Narvai PC, Frazão P, Roncalli AG, Antunes JLF. Cárie dentária no Brasil: declínio, polarização, iniqüidade e exclusão social. Rev Panam Salud Publica 2006; 19(6):385-393.

14. Ferreira-Júnior OM, Freire MC, Moreira RS, Costa LR. Contextual and individual determinants of dental pain in preschool children. Community Dent Oral Epidemiol 2015; 43(4):349-356.

15. Peres MA, Peres KG, Frias AC, Antunes JL. Contextual and individual assessment of dental pain period prevalence in adolescents: a multilevel approach. $B M C$ Oral Health 2010; 10:20.

16. Powers LJ, Grana JR, Keen ND, Hanchak NA. Preventive service utilization as a predictor for emergency dental examinations. Community Dent Health 2000; 17(1):20-23.

17. Silva K, Kunzel C, Yoon RK. Utilization of emergency services for non-traumatic dental disease. J Clin Pediatr Dent 2013; 38(2):107-112.

18. Singhal A, Momany ET, Jones MP, Caplan DJ, Kuthy RA, Buresh CT, Damiano PC. Dental care after an emergency department visit for dental problems among adults enrolled in Medicaid. J Am Dent Assoc 2016; 147(2):111119.

19. Von Kaenel D, Vitangeli D, Casamassimo PS, Wilson S, Preisch J. Social factors associated with pediatric emergency department visits for caries-related dental pain. Pediatr Dent 2001; 23(1):56-60.
20. Programa das Nações Unidas para o Desenvolvimento (PNUD). Índice de Desenvolvimento Humano Municipal (IDHM) [Internet]. 2013 [acessado 2015 Jun 16]. Disponível em: http://www.pnud.org.br/idh/IDHM.aspx?indiceAccordion $=0 \& l i=l i \_I D H M$

21. Austregésilo SC, Leal MCC, Figueiredo N, Góes PSA. The Interface between Primary Care and Emergency Dental Services (SOU) in the SUS: the interface between levels of care in oral health. Cien Saude Colet 2015; 20(10):31113120.

22. Baldani MH, Mendes YB, Lawder JA, Lara AP, Rodrigues $\mathrm{MM}$, Antunes JL. Inequalities in dental services utilization among Brazilian low-income children: the role of individual determinants. J Public Health Dent 2011; 71(1):46-53.

23. Baldani ML, Pupo YM, Lawder JAC, Silva FFM, Antunes JLF. Determinantes Individuais da Utilização Recente de Serviços Odontológicos por Adolescentes e Adultos Jovens de Baixa Renda. Pesq Bras Odontoped Clin Integr 2011; 11(1):91-98.

24. Harnagea H, Couturier Y, Shrivastava R, Girard F, Lamothe L, Bedos CP, Emami E Barriers and facilitators in the integration of oral health into primary care: a scoping review. BMJ Open 2017; 7(9):e016078.

25. Machado GCM, Daher A, Costa LR. Factors associated with no dental treatment in preschoolers with toothache: A cross-sectional study in outpatient public emergency service. Int J Environ Res Public Health 2014; 11(8):80588068.

26. Vargas CM, Ronzio CR. Disparities in early childhood caries. BMC Oral health 2006; 6(Supl. 1):S3.

27. Badri P, Saltaje H, Flores-Mir C, Amin M. Factors affecting children's adherence to regular dental attendance: a systematic review. J Am Dent Assoc 2014; 145(8):817-828.

28. Silva ZP, Ribeiro MCSA, Barata RB, Almeida MF. Perfil sociodemográfico e padrão de utilização dos serviços de saúde do Sistema Único de Saúde (SUS), 2003- 2008. Cien Saude Colet 2011; 16(9):3807-3816.

29. Brasil. Ministério da Saúde (MS). Politica Nacional de Atenção Básica. Diretrizes da política nacional de saúde bucal [Internet]. Brasília: MS; 2004 [acessado 2015 Jun 16]. Disponível em: http://189.28.128.100/dab/docs/publicacoes/geral/diretrizes_da_politica_nacional_de_saude_bucal.pdf

30. Brito GEG, Mendes ACG, Santos Neto PM. Purpose of work in the Family Health Strategy. Interface (Botucatu) 2018; 22(64):77-86.

Artigo apresentado em 31/08/2018

Aprovado em 19/01/2019

Versão final apresentada em 21/01/2019 